\title{
SPARSE MODAL ESTIMATION OF 2-D NMR SIGNALS
}

\author{
Souleymen Sahnoun, El-Hadi Djermoune and David Brie \\ CRAN, Université de Lorraine, CNRS \\ Boulevard des Aiguillettes, BP 70239, 54506 Vandoeuvre, France \\ firstname. lastnameduniv-lorraine. fr
}

\begin{abstract}
We propose a sparse modal estimation approach for analyzing 2-D NMR signals. It consists in decomposing the 2-D problem into two 1-D modal estimations. Each 1-D problem is formulated in terms of simultaneous sparse approximation which is efficiently solved using the Simultaneous Orthogonal Matching Pursuit method associated with a multi-grid dictionary refinement. Then, we propose a new criterion for mode pairing which comes down to solve a sparse approximation problem involving a low dimensional dictionary. The effectiveness of the method is demonstrated on real NMR data.
\end{abstract}

Index Terms - Modal retrieval, sparse approximation, multi-grid, 2-D NMR

\section{INTRODUCTION}

Nuclear magnetic resonance (NMR) spectroscopy is a powerful tool for the determination of chemical structures and molecular interactions [1]. The multidimensional $(R-\mathrm{D})$ NMR spectroscopy enables the detection and interpretation of interactions that are impossible to analyze along a single dimension which makes it essential for the study of macromolecular structures [2]. Data provided by the spectroscope are signals corresponding to the relaxation of the nuclear magnetization; these signals can be modeled by a superposition of damped complex sinusoids. The problem consists in the determination of the multidimensional signal frequencies and decaying rates. In this paper, we focus on 2-D signals.

This is a classical problem in signal processing and several modal retrieval approaches have been already proposed for 2-D signal parameters estimation. For example, we can cite subspace- and linear prediction-based approaches such as TLS-Prony [3], matrix enhancement and matrix pencil (MEMP) [4], 2-D ESPRIT [5], improved multidimensional folding (IMDF) [6], etc. Some of these methods (TLS-Prony and MEMP) separately estimate the modes (including frequencies and damping factors) of each dimension and then use matching algorithms in order to form the pairs of modes. The matching criterion used in MEMP fails in presence of identical modes in one or more dimensions. To overcome this problem, other methods perform a joint diagonalization of two matrices by introducing a weighting parameter $[5,7]$ to avoid rank deficiency. For all these methods, it is necessary to select a priori the correct number of modes that have to be estimated. This information is often not available in real applications such as in NMR spectroscopy. Recently, methods based on sparse approximations have been proposed to address the harmonic or modal retrieval problem $[8,9,10]$. Most of these methods are intended for 1-D signals. They share the advantage that their use does not require necessarily prior knowledge of the number of modes, but their performances depend on a good grid selection allowing the construction of the dictionary [11]. Clearly, to achieve a good resolution, it is necessary to select a fine grid of frequencies and damping factors leading to a large dictionary. This complexity is further increased in the case of 2-D signals in which we are confronted with 2-D grids. We have recently proposed a multi-grid sparse approximation method [12] where the estimation is started with a coarse grid which is then iteratively refined by inserting new grid points leading to a multiresolution-like scheme. For 1-D signals, this method allows achieving a good resolution while maintaining reasonable the dictionary size. However, for large 2-D NMR signals, this is often not sufficient. To deal with such signals, we propose here a 2-D sparse estimation method based on two main steps. In the first step, the method estimates the modes separately in each dimension by using the simultaneous sparse approximation principle [13] combined with the multi-grid approach. Then, in the second step, the estimated modes are paired. The new pairing procedure also calls for the resolution of a sparse approximation problem where the dictionary is built from the estimated 1-D modes and hence only includes few columns. Consequently, the computational complexity of the multi-grid approach is significantly reduced, which allows its application to spectroscopy signals.

The remainder of this paper is organized as follows. In section 2, we present the problem statement and the formulation of the 2-D modal estimation problem as two simultaneous sparse estimation problems followed by a new procedure for pairing 2-D modes. The effectiveness of the proposed method is demonstrated using simulation and experimental signals in sections 3 and 4 respectively. 


\section{SIMULTANEOUS SPARSE APPROXIMATION FOR 2-D MODAL SIGNALS}

\subsection{Signal model and data filtering}

The classical model for 2-D NMR signals is the superposition of 2-D exponentially decaying sinusoids in noise. In symbols:

$$
\tilde{y}\left(m_{1}, m_{2}\right)=\sum_{i=1}^{F} c_{i} a_{i}^{m_{1}-1} b_{i}^{m_{2}-1}+e\left(m_{1}, m_{2}\right)
$$

for $m_{1}=1, \ldots, M_{1}$ and $m_{2}=1, \ldots, M_{2}$, where $a_{i}=$ $e^{-\alpha_{a, i}+j 2 \pi f_{a, i}}$ are the modes of the first dimension and $b_{i}=e^{-\alpha_{b, i}+j 2 \pi f_{b, i}}$ are those of the second dimension. $\left\{\alpha_{a, i}, \alpha_{b, i}\right\}_{i=1}^{F}$ are damping factors, $\left\{f_{a, i}, f_{b, i}\right\}_{i=1}^{F}$ are frequencies and $\left\{c_{i}\right\}_{i=1}^{F}$ are complex amplitudes; $e\left(m_{1}, m_{2}\right)$ is an additif noise. The problem is to estimate the set of parameters $\left\{a_{i}, b_{i}, c_{i}\right\}_{i=1}^{F}$ from the observed signal $\tilde{y}\left(m_{1}, m_{2}\right)$. In this paper, the tilde symbol $\left(^{\sim}\right)$ denotes a noisy signal.

Let $\mathbf{Y}$ be the noise-free data matrix containing the samples $y\left(m_{1}, m_{2}\right)$. Then, $\mathbf{Y}$ may be written as:

$$
\begin{aligned}
& \mathbf{Y}=\left[\mathbf{y}_{1}, \mathbf{y}_{2}, \cdots, \mathbf{y}_{M_{2}}\right] \\
& =\left[\begin{array}{llll}
\sum_{i=1}^{F} c_{i} \mathbf{a}_{i} & \sum_{i=1}^{F} c_{i} b_{i} \mathbf{a}_{i} & \cdots & \sum_{i=1}^{F} c_{i} b_{i}^{M_{2}-1} \mathbf{a}_{i}
\end{array}\right]
\end{aligned}
$$

where $\mathbf{a}_{i}=\left[1, a_{i}, \ldots, a_{i}^{M_{1}-1}\right]^{T}$. By putting $c_{i}^{\prime}\left(m_{2}\right)=$ $c_{i} b_{i}^{m_{2}-1}$, the columns $\mathbf{y}_{m_{2}}$ of $\mathbf{Y}$ can be considered as multiple experiences involving the same signal generated by the modes $a_{i}, i=1, \ldots, F$, but with different amplitudes for each experience. Then, the noisy matrix $\tilde{\mathbf{Y}}$ can be written as:

$$
\tilde{\mathbf{Y}}=\mathbf{A H}+\mathbf{E}
$$

where $\mathbf{A}$ is a Vandermonde matrix whose columns are $\left\{\mathbf{a}_{i}\right\}_{i=1}^{F}, \mathbf{H}$ is a $F \times M_{2}$ matrix with entries $c_{i}^{\prime}\left(m_{2}\right)$, and $\mathbf{E}$ is the matrix formed with $e\left(m_{1}, m_{2}\right)$. To reduce the noise influence, we follow [9] by replacing $\tilde{\mathbf{Y}}$ by its SVD-based low rank approximation $\mathbf{Y}$.

\subsection{Simultaneous sparse estimation}

From (2), it is easy to see that the modes $\left\{a_{i}\right\}$ can be obtained from any column of $\mathbf{Y}$. Similarly, these modes may be estimated from any column of the filtered matrix $\breve{\mathbf{Y}}$ but, in order to reduce the estimation variance, we will use all the columns. Hence, for each column $\breve{\mathbf{y}}_{m_{2}}, m_{2}=1, \ldots, M_{2}$, the modal estimation problem can be formulated as a sparse approximation which is solution of the following constrained optimization problem:

$$
\mathbf{x}_{m_{2}}=\min _{\mathbf{x}}\|\mathbf{x}\|_{0} \quad \text { subject to } \quad\left\|\breve{\mathbf{y}}_{m_{2}}-\mathbf{Q}_{a} \mathbf{x}\right\|_{2}^{2} \leq \epsilon
$$

where $\mathbf{Q}_{a}$ is a modal dictionary and $\mathbf{x}$ is a (sparse) vector containing the coefficients of the activated columns in $\mathbf{Q}_{a}$, the latter being estimates of signals $\mathbf{a}_{i}$. The dictionary $\mathbf{Q}_{a}$ can be defined as follows. Let $\alpha_{\max }$ be an upper bound on $\left\{\alpha_{a, i}\right\}_{i=1}^{F}$ and let $P$ the number of points of a uniform grid covering the damping factor interval $\left[0, \alpha_{\max }\right]$. Similarly, let $K$ be the number of points of a uniform grid covering the frequency interval $\left[0,1\left[\right.\right.$. Then $\mathbf{Q}_{a}$ is given by

$$
\begin{array}{r}
\mathbf{Q}_{a}=\left[\mathbf{q}(0,0), \ldots, \mathbf{q}\left(0,(K-1) \delta_{f}\right), \mathbf{q}\left(\delta_{\alpha}, 0\right), \ldots,\right. \\
\left.\mathbf{q}\left(\delta_{\alpha},(K-1) \delta_{f}\right), \ldots, \mathbf{q}\left((P-1) \delta_{\alpha},(K-1) \delta_{f}\right)\right]
\end{array}
$$

where $\mathbf{q}(\alpha, f)=\mathbf{a}(\alpha, f) /\|\mathbf{a}(\alpha, f)\|_{2}$ with $\mathbf{a}(\alpha, f)=$ $\left[1, e^{(-\alpha+j 2 \pi f)}, \ldots, e^{(-\alpha+j 2 \pi f)\left(M_{1}-1\right)}\right]^{T}, \delta_{\alpha}=\alpha_{\max } / P$, and $\delta_{f}=1 / K$. In short, $\mathbf{Q}_{a}$ is obtained from a discretization of the $(\alpha, f)$ plane. Each point of the grid corresponds to a hypothetic mode. The total number of columns of $\mathbf{Q}_{a}$ is $N=K P \gg F$, each of them is called atom. The fact that each vector $\breve{\mathbf{y}}_{m_{2}}$ corresponds to a 1-D signal generated by the same modes implies that the positions of non-zero coefficients should be the same in all sparse vectors $\mathbf{x}_{m_{2}}$, $m_{2}=1, \ldots, M_{2}$. This property may be advantageously exploited using simultaneous sparse estimation which is a particular group sparsity approach. The main interest of the simultaneous sparse estimation is to decrease the estimation variance as compared to the independent resolution of (4) for all values of $m_{2}$. To do so, let $\mathbf{X}=\left[\mathbf{x}_{1}, \mathbf{x}_{2}, \ldots, \mathbf{x}_{M_{2}}\right]$, then the sparsity of $\mathbf{X}$ may be measured by computing the Euclidian norm of each row; those providing a non-zero norm define the positions of the activated atoms in the dictionary $\mathbf{Q}_{a}$. Therefore, we are facing a simultaneous sparse approximation problem:

$$
\min _{\mathbf{X}}\|\mathbf{X}\|_{2,0} \quad \text { subject to } \quad\left\|\breve{\mathbf{Y}}-\mathbf{Q}_{a} \mathbf{X}\right\|_{f}^{2} \leq \epsilon
$$

where

$$
\begin{aligned}
& \left\|\breve{\mathbf{Y}}-\mathbf{Q}_{a} \mathbf{X}\right\|_{f}^{2}=\left\|\operatorname{vec}\left(\breve{\mathbf{Y}}-\mathbf{Q}_{a} \mathbf{X}\right)\right\|_{2}^{2} \\
& \|\mathbf{X}\|_{2,0}=\left\|\left[\begin{array}{lll}
\|\mathbf{X}[1,:]\|_{2} & \cdots & \|\mathbf{X}[N,:]\|_{2}
\end{array}\right]^{T}\right\|_{0}
\end{aligned}
$$

and $\mathbf{X}[n,:]$ stands for the $n$th row of $\mathbf{X}$. Several works have been already presented to solve the simultaneous sparse approximation problem involving several signals. A survey of these algorithms is presented in [14]. In this paper we use the Simultaneous Orthogonal Matching Pursuit method (SOMP) [13]. Once the modes corresponding to the first dimension $\left(a_{i}\right)$ are estimated, those of the second one $\left(b_{k}\right)$ are obtained in the same manner by processing the matrix $\tilde{\mathbf{Y}}^{T}$ and using a dictionary $\mathbf{Q}_{b}$. Finally, $a_{i}$ and $b_{k}$ have to be paired to form the 2-D modes. This is addressed in section 2.4 but, before, to reach a good resolution, we propose to associate to the simultaneous sparse approximation a multi-grid dictionary refinement.

\subsection{Multi-grid dictionary refinement}

To achieve a high-resolution modal estimation, a possible way is to define a high resolution dictionary often resulting 


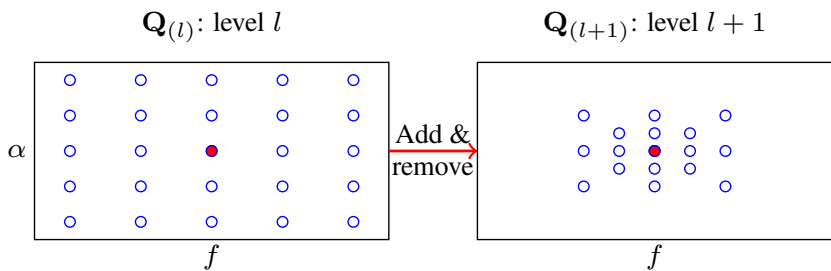

Fig. 1. The multi-grid dictionary refinement. (०) Dictionary atoms. (•) Activated atoms.

in a prohibitive computational burden. Rather, we propose to start with a coarse one ( $N$ low) and to adaptively refine it through a multi-grid scheme. This principle proposed in [12] is sketched on figure 1 . The main idea is the adaptation of the dictionary as a function of the previous dictionary and the estimated coefficients. Let $l$ be the current grid level $(l=0, \ldots, L-1)$. At level $l$, we first restore the signal $\mathbf{X}_{(l)}$ related to the dictionary $\mathbf{Q}_{(l)}$ by applying the S-OMP method, where $\mathbf{Q}_{(l)}$ relates to either $\mathbf{Q}_{a}$ or $\mathbf{Q}_{b}$ at level $l$. Then we refine the dictionary by inserting atoms in between pairs of $\mathbf{Q}_{(l)}$, in the neighborhood of each activated atom while removing the non-active ones and we apply again the S-OMP method at level $l+1$ to restore $\mathbf{X}_{(l+1)}$ with respect to the refined dictionary $\mathbf{Q}_{(l+1)}$. Thus we refine iteratively the dictionary until the maximum level $l=L-1$ is reached.

\subsection{2-D modes pairing}

The 2-D signal model (1) can be rewritten as:

$$
\tilde{\mathbf{y}}=(\mathbf{A} \odot \mathbf{B}) \mathbf{c}+\mathbf{e}
$$

where $\tilde{\mathbf{y}}=\operatorname{vec}\left(\tilde{\mathbf{Y}}^{T}\right), \mathbf{A} \in \mathbb{C}^{M_{1} \times F}$ and $\mathbf{B} \in \mathbb{C}^{M_{2} \times F}$ are Vandermonde matrices whose columns are $\mathbf{a}_{i}$ and $\mathbf{b}_{k}$, respectively. The vector $\mathbf{c}=\left[c_{1}, \ldots, c_{F}\right]^{T}$ gathers the 2-D mode amplitudes and $\odot$ stands for the Khatri-Rao product. The method we propose here for pairing of 2-D modes consists in exploiting the sparse approximation principle for $R$-D signals [12]. We denote by $F_{a}$ and $F_{b}$ the number of estimated modes $\hat{a}_{i}$ and $\hat{b}_{k}$, respectively. We define the two dictionaries $\hat{\mathbf{Q}}_{a}$ and $\hat{\mathbf{Q}}_{b}$ as follows:

$$
\begin{aligned}
\hat{\mathbf{Q}}_{a} & =\left[\hat{\mathbf{a}}_{1}, \hat{\mathbf{a}}_{2}, \ldots, \hat{\mathbf{a}}_{F_{a}}\right] \\
\hat{\mathbf{Q}}_{b} & =\left[\hat{\mathbf{b}}_{1}, \hat{\mathbf{b}}_{2}, \ldots, \hat{\mathbf{b}}_{F_{b}}\right]
\end{aligned}
$$

where $\hat{\mathbf{a}}_{i}=\left[1, \hat{a}_{i}, \ldots, \hat{a}_{i}^{M_{1}-1}\right]^{T}$ for $i=1, \ldots, F_{a}$ and $\hat{\mathbf{b}}_{k}=$ $\left[1, \hat{b}_{k}, \ldots, \hat{b}_{k}^{M_{2}-1}\right]^{T}$ for $k=1, \ldots, F_{b}$. Afterwards, using the Kronecker product, we form the dictionary $\hat{\mathbf{Q}}$ defined by:

$$
\hat{\mathbf{Q}}=\hat{\mathbf{Q}}_{a} \otimes \hat{\mathbf{Q}}_{b} \text {. }
$$

In order to select the pairs of 2-D modes present in $\breve{\mathbf{y}}$, we solve the sparse problem

$$
\min _{\mathbf{x}}\|\mathbf{x}\|_{0} \quad \text { subjet to } \quad\|\breve{\mathbf{y}}-\hat{\mathbf{Q}} \mathbf{x}\|^{2} \leq \epsilon .
$$

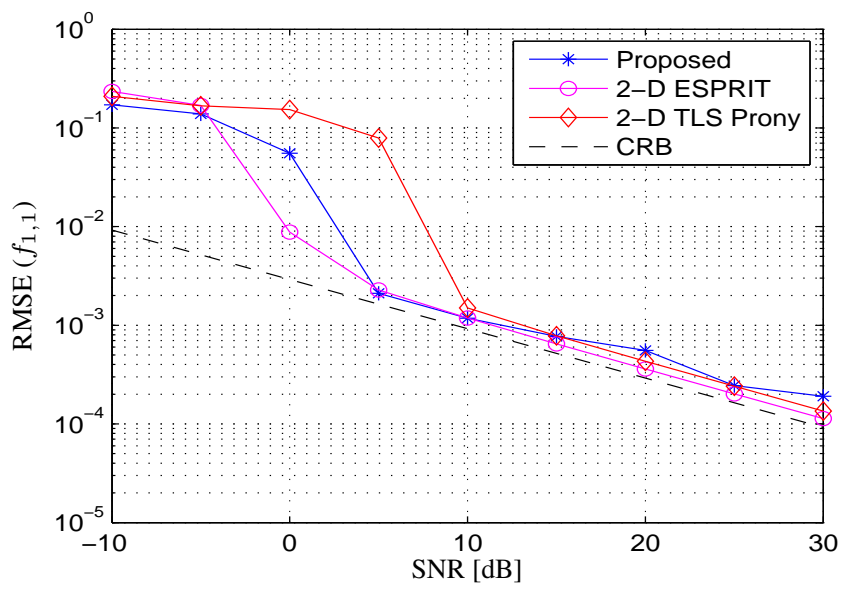

Fig. 2. Simulation result: root-mean square error of $f_{1,1}$.

This problem is solved using a greedy method such as OMP or SBR [15]. Note that this pairing algorithm is efficient even in presence of identical modes in one or both dimensions.

\section{ALGORITHM AND PERFORMANCES}

The complete algorithm can be summarized as follows:

1. Perform the SVD of $\tilde{\mathbf{Y}}$ and take its low rank approximation $\breve{Y}$.

2. Apply the multi-grid algorithm combined with S-OMP on matrix $\breve{Y}$ to obtain the modes $a_{i}$ (first dimension).

3. Repeat step 2 using $\breve{\mathbf{Y}}^{T}$ to estimate the modes $b_{i}$ (second dimension).

4. Form the 2-D modes using the pairing procedure.

The performances of the proposed method are assessed by numerical simulations and compared to those obtained with 2D TLS-Prony and 2-D ESPRIT which is one of the most efficient 2-D modal estimation approaches. The simulated signal of size $30 \times 30$ samples contains three modes:

$$
\begin{aligned}
& \left(f_{1,1}, \alpha_{1,1} ; f_{1,2}, \alpha_{1,2}\right)=(0.164,0.080 ; 0.342,0.075), \\
& \left(f_{2,1}, \alpha_{2,1} ; f_{2,2}, \alpha_{2,2}\right)=(0.310,0.075 ; 0.110,0.050), \\
& \left(f_{3,1}, \alpha_{3,1} ; f_{3,2}, \alpha_{3,2}\right)=(0.412,0.050 ; 0.090,0.243) .
\end{aligned}
$$

All the amplitudes are set to 1 . We perform Monte Carlo simulations with different SNR levels. For the proposed method, the initial grid used to build the dictionary is the same for both dimensions; it contains 40 frequency points uniformly distributed over the interval [0,1[, and 4 damping factors $\alpha \in$ $\{0,0.025,0.05,1\}$. We perform 30 levels of resolution for each estimation. The RMSE of the frequency $f_{1,1}$ is presented in figure 2. We observe that the proposed method gives satisfactory results since it reaches performance levels comparable to that of 2-D ESPRIT, which are better than 2-D TLS-Prony at low SNR. It is worth noticing that the proposed approach is able to handle large 2-D signals; 2-D ESPRIT does not, which precludes its use for large 2-D NMR data processing. 


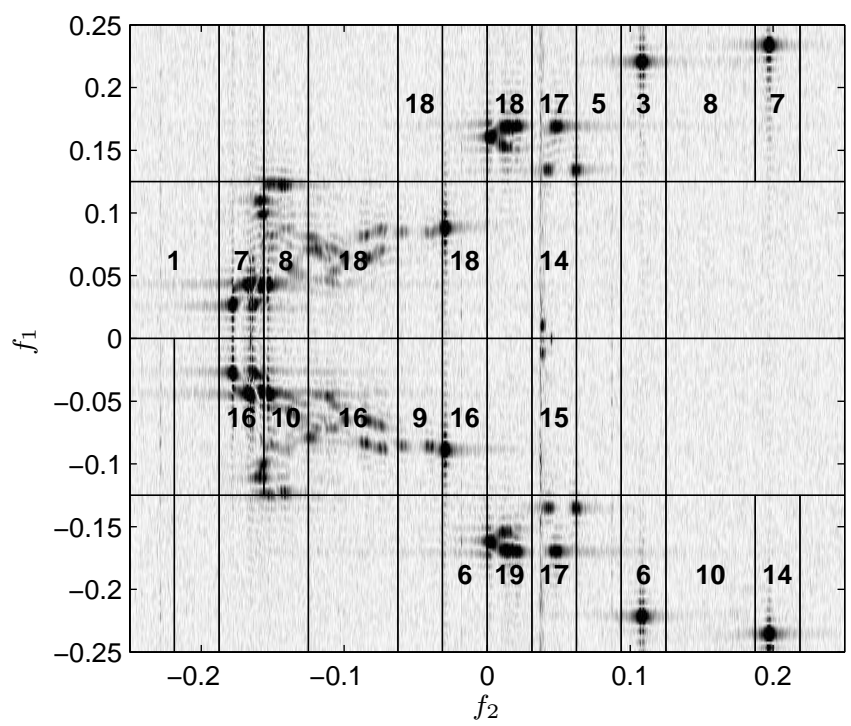

Fig. 3. Results achieved by the proposed method in the spectral region $[-0.25,0.25] \times[-0.25,0.25]$ of the experimental 2-D NMR signal using a sub-band decomposition. The number of estimated modes is indicated in each sub-band.

\section{2-D NMR SIGNAL ANALYSIS}

The analyzed signal is of dimension $64 \times 2024$. Before applying the multigrid approach combined with S-OMP algorithm, we decompose the signal into sub-bands according to the approach presented in [16]. We use the same initial grid as before. The number of resolution levels is set to 30. Figure 3 shows the final decomposition into sub-bands of the 2-D NMR signal in the spectral range $[-0.25,0.25] \times[-0.25,0.25]$ as well as the number of modes estimated in each sub-band by the proposed method. The results obtained in certain sub-bands are shown in figure 4 where the positions of estimated modes are indicated by circles. We observe that the proposed method performs well in the case of close and/or aligned modes. Moreover, the method proves to be competitive as compared to 2-D TLSProny $[17,18]$ in terms of estimation accuracy and computational burden.

\section{CONCLUSION}

In this paper we proposed a sparse 2-D modal estimation approach suited to large 2-D NMR signals. The idea consists in exploiting the simultaneous sparse approximation principle to separate this joint estimation problem into two 1-D problems. The modes are then coupled using a new algorithm which is also based on the minimization of a sparse criterion. Finally, tests performed on 2-D NMR signals showed the potential of the sparse multi-grid methods. As a future work, this method will be extended for multidimensional $(R>2)$ signals.



(a) Sub-band $(0.12,0.24) \times(0.095,0.12)$

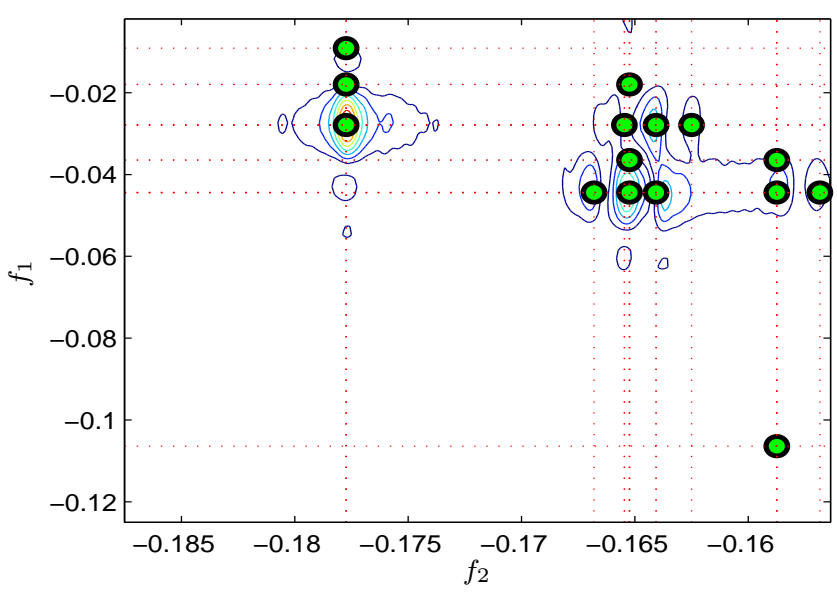

(b) Sub-band $(-0.12,0.0) \times(-0.175,0.155)$

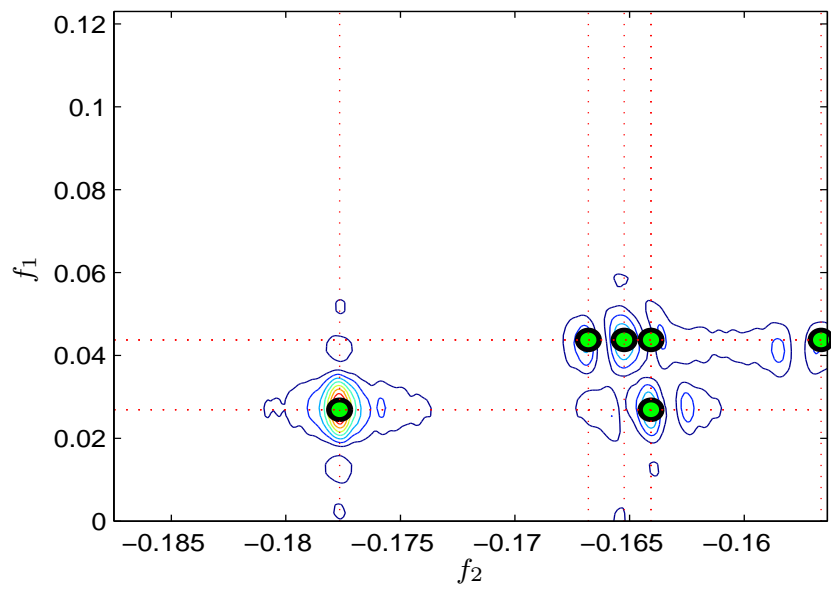

(c) Sub-band $(0.0,0.12) \times(-0.175,0.155)$

Fig. 4. Results achieved in some sub-bands of the experimental 2-D NMR signal. (o) Estimated modes. (...) Mode coordinates.

\section{REFERENCES}

[1] J. C. Hoch and A. Stern, NMR data processing, WileyLiss, NY, 1996. 
[2] A. Bax and L. Lerner, "Two-dimensional nuclear magnetic resonance spectroscopy," Science, vol. 232, pp. 960-967, May 1986.

[3] J. J. Sacchini, W. M. Steedly, and R. L. Moses, "Twodimensional Prony modeling and parameter estimation," IEEE Trans. Signal Process., vol. 41, no. 11, pp. $3127-$ 3137, 1993.

[4] Y. Hua, "Estimating two-dimensional frequencies by matrix enhancement and matrix pencil," IEEE Trans. Signal Process., vol. 40, no. 9, pp. 2267-2280, 1992.

[5] S. Rouquette and M. Najim, "Estimation of frequencies and damping factors by two-dimensional ESPRIT type methods," IEEE Trans. Signal Process., vol. 49, no. 1, pp. 237-245, 2001.

[6] J. Liu and X. Liu, "An eigenvector-based approach for multidimensioanl frequency estimation with impoved identifiability," IEEE Trans. Signal Process., vol. 54, no. 12, pp. 4543-4556, decembre 2006.

[7] J. Liu, X. Liu, and X. Ma, "Multidimensional frequency estimation with finite snapshots in the presence of identical frequencies," IEEE Trans. Signal Process., vol. 55, pp. 5179-5194, 2007.

[8] M. M. Goodwin and M. Vetterli, "Matching pursuit and atomic signal models based on recursive filter banks," IEEE Trans. Signal Process., vol. 47, no. 7, pp. 1890$1902,1999$.

[9] D. Malioutov, M. Cetin, and A. S. Willsky, "A sparse signal recontruction perspective for source localization with sensor arrays," IEEE Trans. Signal Process., vol. 53, no. 8, pp. 3010-3022, 2005.

[10] P. Stoica, P. Babu, and J. Li, "Spice: A sparse covariance-based estimation method for array processing," IEEE Trans. Signal Process., vol. 59, no. 2, pp. 629-638, 2011.

[11] P. Stoica and P. Babu, "Sparse estimation of spectral lines: grid selection problems and their solutions," IEEE Trans. Signal Process., vol. 60, no. 2, pp. 962-967, 2012.

[12] S. Sahnoun, E.-H. Djermoune, C. Soussen, and D. Brie, "Sparse multidimensional modal analysis using a multigrid dictionary refinement," EURASIP J. Adv. Signal Process., March 2012.

[13] J.A. Tropp, A.C. Gilbert, and M.J. Strauss, "Algorithms for simultaneous sparse approximation. Part I: Greedy pursuit," Signal Processing, vol. 86, no. 3, pp. 572-588, 2006.
[14] A. Rakotomamonjy, "Surveying and comparing simultaneous sparse approximation (or group-Lasso) algorithms," Signal processing, vol. 91, no. 7, pp. 15051526, 2011.

[15] C. Soussen, J. Idier, D. Brie, and J. Duan, "From Bernoulli-Gaussian deconvolution to sparse signal restoration," IEEE Trans. Signal Process., vol. 56, no. 10, pp. 4572-4584, 2011.

[16] E. H. Djermoune, G. Kasalica, and D. Brie, "Estimation of the parameters of two-dimensional NMR spectroscopy signals using an adapted subband decomposition," in Proc. ICASSP, Las Vegas, USA, 2008, pp. 3641-3644.

[17] E. H. Djermoune, D. Brie, and M. Tomczak, "A subband algorithm for estimating the parameters of twodimensional exponential signals," in Proc. European Signal Process. Conf., Glasgow, Scotland, 2009, pp. 1032-1036.

[18] E. H. Djermoune, M. Tomczak, and D. Brie, "NMR data analysis: A time-domain parametric approach using adaptive subband decomposition," OGST - Rev. IFP Energies Nouvelles, 2012, (to appear). 